\title{
MULTIPLE VERTEBRAL FRACTURES SECONDARY TO IRREGULAR USE OF DENOSUMAB
}

Claudia Valeria Vierhout (Hospital PUC Campinas, Campinas, SP, Brasil), Nadia Regina Bossolan Schincariol (Hospital PUC Campinas, Campinas, SP, Brasil), José Alexandre Mendonça (Hospital PUC Campinas, Campinas, SP, Brasil), Lucas Eduardo Pedri (Hospital PUC Campinas, Campinas, SP, Brasil), Andre Marun Lyrio (Hospital PUC Campinas, Campinas, SP, Brasil), Rubens Bonfiglioli (Hospital PUC Campinas, Campinas, SP, Brasil), José Roberto Provenza (Hospital PUC Campinas, Campinas, SP, Brasil), Vanessa Ramos Guissa (Hospital PUC Campinas, Campinas, SP, Brasil), Fernanda Bertucci Sanches (Hospital PUC Campinas, Campinas, SP, Brasil), Thais Campos Ferreira Pinto (Hospital PUC Campinas, Campinas, SP, Brasil), Igor Tadeu Garcia Ferreira (Hospital PUC Campinas, Campinas, SP, Brasil)

\section{BACKGROUND}

Denosumab is a fully monoclonal antibody used to treat osteoporosisby blocking the binding between a receptor on the osteoclast surface (RANK) and its cytokine (RANKL), inihibiting osteoclast formation, function and survival. Consequently, bone resorption slows, decreasingthe risk of bone fractures. Despite of that, inadvertent discontinuation of the drug may increase the risk of fractures. It is known that denosumab does not incorporate into bone matrix. That is why, after suspended, its effects are reversible. Even further, a severe rebound effect may occour - with rapid increases in bone resorption- if a medication dose is delayed for a few months, putting the patient in a raised risk of bone fracture. The following case report is about a patient who progressively delayed doses of denosumab, culminating in extensive vertebral fractures.

\section{CASE REPORT}

A 57-year-old woman with history of osteoporosis was in use of denosumab for the last 3 years. In the first year of treatment, patient made regular use of the medication, respecting the correct interval between doses. In the next year, there were consecutive delays in administering the drug (1 to 2 months late). After a 9 months pause, patient presented acute inflammatory low back pain. There was no report of trauma. Magnetic resonance imaging revealed vertebral collapses in T6,T7,T8,T9,T10, L1 and L2. As described in literature, patient presented fragility fractures after irregular use of denosumab.

\section{CONCLUSION}

Denosumab is an important and effective option in the treatment of osteoporosis. The drug discontinuation, on the other hand, even if for a short period, may result in bone loss and structural damage. The rheumatologist should alert the patient to this fact and maintain strict control of the medication schedule. If irregular use of denosumab is noticed, a bisphosphonate or another antiresorptiveagent should be promptly initiated. 